\title{
Planar Hall Effect in Ferromagnetic (Ga,Mn)As/GaAs Superlattices
}

\author{
W. Wesela ${ }^{a, b}$, T. WOSIŃSKi ${ }^{a}$, A. MA̧KOSA $^{a}$, T. Figielski ${ }^{a}$, \\ J. SADOWSKI ${ }^{a, c, d}$, F. TERKI ${ }^{d}$ AND S. ChARAR ${ }^{d}$ \\ ${ }^{a}$ Institute of Physics, Polish Academy of Sciences \\ al. Lotników 32/46, 02-668 Warsaw, Poland \\ ${ }^{b}$ College of Science, Cardinal S. Wyszyński University, Warsaw, Poland \\ ${ }^{c}$ MAX-Lab, Lund University, 22100 Lund, Sweden \\ ${ }^{d}$ Groupe d'Etude des Semiconducteurs CC074, Université Montpellier II \\ Montpellier, France
}

\begin{abstract}
The planar Hall effect was used for investigation of magnetic anisotropy in short period $(\mathrm{Ga}, \mathrm{Mn}) \mathrm{As} / \mathrm{GaAs}$ superlattices epitaxially grown on (001) oriented GaAs substrate. The results confirmed the existence of low-temperature magnetocrystalline anisotropy in the superlattices with the easy magnetic axes directed along the two in-plane $\langle 100\rangle$ directions. Attention is paid to the two-state behaviour of the planar Hall resistance at zero magnetic field that provides its usefulness for applications in non-volatile memory devices.
\end{abstract}

PACS numbers: 73.50.Jt, 75.50.Pp, 75.30.Gw, 85.75.-d

\section{Introduction}

Magnetic properties of ferromagnetic materials strongly affect their electrical-transport properties, which, in turn, can provide an additional insight into the nature of their magnetism. On the other hand, for microstructures fabricated from ferromagnetic materials or dilute magnetic materials, like (Ga,Mn)As thin films, magnetic properties are inherently difficult to measure, due to a small volume of ferromagnetic material which is involved. In these cases magnetotransport measurements with a high signal-to-noise ratio are much simpler than direct measurements of magnetization. In particular, the so-called planar Hall effect (PHE) [1], which manifests itself as a spontaneous transverse voltage that develops, owing to the spin-orbit interaction, in response to longitudinal current flow under an in-plane magnetic field or even in the absence of an applied magnetic field, can 
be of great utility in the study of dilute ferromagnetic semiconductors. Recently, Tang et al. [2] have shown that the magnitude of PHE in (Ga,Mn)As layers can be up to four orders of magnitude greater than the one previously found in metallic ferromagnets, and have referred this effect to as the giant PHE.

$(\mathrm{Ga}, \mathrm{Mn}) \mathrm{As}$ has become a model ferromagnetic semiconductor widely used for studying new physical phenomena and development of novel magnetoelectronic devices prospective for future applications in spin electronics. Homogeneous layers of $\mathrm{Ga}_{1-x} \mathrm{Mn}_{x}$ As containing up to $8 \%$ of $\mathrm{Mn}$ atoms can be grown by a low-temperature molecular beam epitaxy (LT-MBE). When intentionally undoped, the layers are of $p$-type, where Mn atoms, substituting the Ga atoms in GaAs crystal lattice, supply both mobile holes and magnetic moments. Below a magnetic-transition temperature, $T_{\mathrm{C}}$, the layers become ferromagnetic due to the hole-mediated ordering of $\mathrm{Mn}$ spins, that is now well understood within the frames of the mean field Zener model for ferromagnetic semiconductors [3]. The same model also predicts magnetic anisotropy induced by the lattice strain present in epitaxial (Ga,Mn)As layers, with tensile and compressive strain inducing out-of-plane and in-plane magnetization, respectively. The latter case corresponds to (Ga,Mn)As layers grown on GaAs substrate, which additionally display $\langle 100\rangle$ in-plane cubic anisotropy. In addition, they exhibit uniaxial anisotropy between the in-plane $\langle 110\rangle$ directions, which can be as well explained in terms of the Zener model assuming a small trigonal distortion, whose microscopic origin is, however, not known [4]. A very large magnitude of PHE observed in $(\mathrm{Ga}, \mathrm{Mn})$ As results primarily from the combined effects of strong spin-orbit interaction in the valence band of the zinc-blende crystal structure and the large spin polarization of holes in (Ga,Mn)As [2]. Recently, we have demonstrated, by comparing with the magnetization data, that the PHE reproduces the magnetic anisotropy in a single $(\mathrm{Ga}, \mathrm{Mn})$ As layer subjected to post-growth annealing [5].

In this paper we report on our investigation of the planar Hall effect in short period (Ga,Mn)As/GaAs superlattices (SLs) and point out to its memory behaviour.

\section{Experimental results}

Short period $\mathrm{Ga}_{0.95} \mathrm{Mn}_{0.05} \mathrm{As} / \mathrm{GaAs}$ SLs were grown by means of the LT-MBE technique on semi-insulating (001)-oriented GaAs substrate [6]. For comparison, a reference structure consisting of a single $50 \mathrm{~nm}$ thick ferromagnetic $\mathrm{Ga}_{0.95} \mathrm{Mn}_{0.05}$ As layer, grown on GaAs under similar conditions, was investigated. All the results presented in this paper refer to the reference layer and to the SL containing 8 monolayers (MLs) of $(\mathrm{Ga}, \mathrm{Mn}) \mathrm{As}$ and 8 MLs of GaAs spacer and the number of repetitions of the $(\mathrm{Ga}, \mathrm{Mn}) \mathrm{As} / \mathrm{GaAs}$ sequence equal to 200. Magneto-transport measurements were carried out on the Hall bars of $150 \mu \mathrm{m}$ width, aligned along the [110] crystallographic direction, prepared by means of photolithographic patterning and chemical etching, whose image is shown 
in the inset in Fig. 1. Both the longitudinal resistance, $R_{x x}$, and the planar Hall resistance, $R_{x y}$, were measured simultaneously using a low-frequency $(27 \mathrm{~Hz})$ lock-in technique. The measurements were performed for various orientations of the in-plane magnetic field swept in the range of $\pm 1 \mathrm{kOe}$ in the temperature range of $4-20 \mathrm{~K}$, i.e. well below $T_{\mathrm{C}}$, which was $50 \mathrm{~K}$ for the $\mathrm{SL}$ and $68 \mathrm{~K}$ for the reference layer.

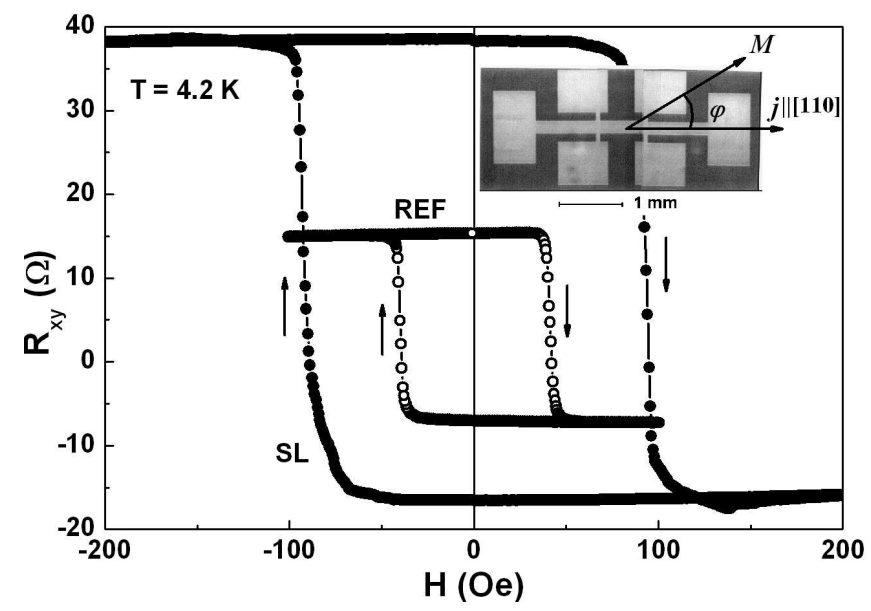

Fig. 1. Planar Hall resistance vs. in-plane magnetic field applied along the [110] crystallographic axis, parallel to the current flow, measured at $4.2 \mathrm{~K}$ for the $\mathrm{Ga}_{0.95} \mathrm{Mn}_{0.05} \mathrm{As} / \mathrm{GaAs}$ superlattice (full symbols) and for the reference $\mathrm{Ga}_{0.95} \mathrm{Mn}_{0.05} \mathrm{As}$ layer (open symbols). The latter data have been vertically offset for clarity. The data measured while sweeping a magnetic field in opposite directions are indicated by arrows. Inset: microscopic image of a Hall bar and the configuration used for PHE measurements.

The investigated structures exhibit a giant magnitude of the PHE. When sweeping the magnetic field, the planar Hall resistance varies non-monotonously alternating its sign and displaying the appearance of a single or double hysteresis loops, depending on the magnetic field orientation and on the sweeping range of the magnetic field $[2,5]$. The dependence of the planar Hall resistance on the magnetic field applied parallel to the current flow, i.e. along the [110] direction, is shown in Fig. 1 for the two structures investigated. While sweeping a magnetic field in a narrow range of \pm 200 Oe, $R_{x y}$ displays a hysteresis loop analogous to the magnetization hysteresis loop. This hysteretic behaviour of the planar Hall resistance, which can assume one of the two stable values of opposite sign at zero magnetic field, that depends on the previously applied field, may be applicable in memory elements. Figure 2 presents such a memory behaviour of the SL planar Hall resistance while switching the magnetic field on and off at either its positive or negative direction along the [110] axis. Very recently we have demonstrated 


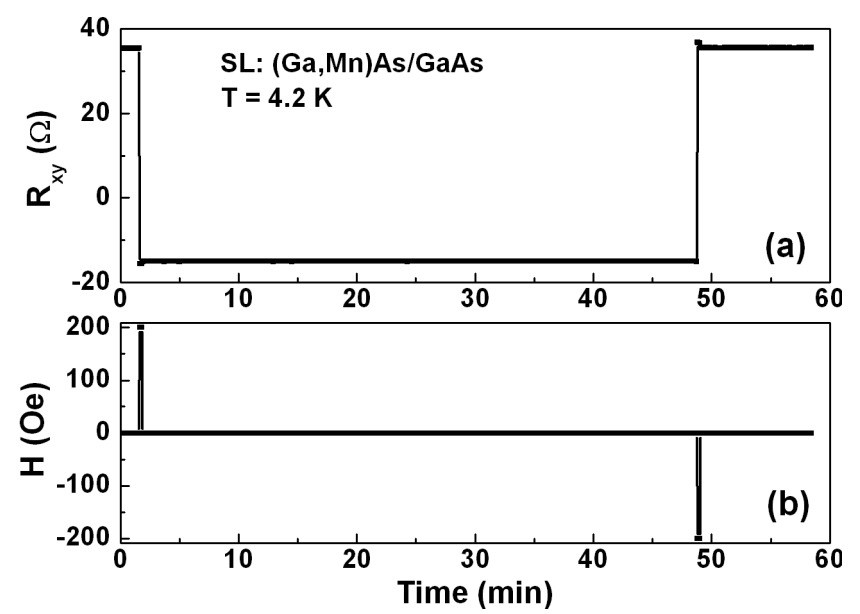

Fig. 2. Planar Hall resistance of the superlattice (a) vs. time while switching the magnetic field (b) on and off at either positive or negative direction along the [110] axis.

another type of memory effect, which appears in magnetoresistance of a three-arm nanostructure fabricated from a ferromagnetic (Ga,Mn)As layer [7].

\section{Discussion and conclusions}

The PHE in magnetic conductors occurs when the resistivity depends on the angle $\varphi$ between directions of the current density $\boldsymbol{j}$ and the magnetization $\boldsymbol{M}$, an effect known as transverse anisotropic magnetoresistance (AMR) [8]. The electric field within a single domain ferromagnetic layer with in-plane magnetization can be written as

$$
\begin{aligned}
& E_{x}=j \rho_{\perp}+j\left(\rho_{\|}-\rho_{\perp}\right) \cos ^{2} \varphi \\
& E_{y}=j\left(\rho_{\|}-\rho_{\perp}\right) \sin \varphi \cos \varphi
\end{aligned}
$$

where the current density is assumed to be along the Hall bar, i.e. along the $x$ axis, $\rho_{\|}$and $\rho_{\perp}$ are resistivities for magnetization vector oriented parallel and perpendicular to the current. The AMR phenomenon is described by Eq. (1) while the transverse resistance $R_{x y}$, i.e. the planar Hall resistance, is expressed by Eq. (2). According to the latter equation $R_{x y}$ exhibits extremum values at the angles $\varphi$ of $45^{\circ}, 135^{\circ}, 225^{\circ}$, and $315^{\circ}$, which correspond to the orientations of the magnetization vector along the in-plane $\langle 100\rangle$ crystallographic directions.

The two-state behaviour of the planar Hall resistance, shown in Figs. 1 and 2 , indicates that the magnetic field scan along the [110] direction results in $90^{\circ}$ rotations of the magnetization vector between two in-plane $\langle 100\rangle$ directions, thus showing that in the both structures investigated, at low temperatures, the cubic [100] and [010] directions represent equivalent easy axes, while both the [110] and $[\overline{1} 10]$ directions correspond to the in-plane hard axes of magnetization. The rigid transitions of the SL planar Hall resistance, occurring in a narrow range of 
magnetic field, evidence that the magnetization vectors of all the magnetic layers constituting the SL rotate together. This result is in line with the existence of a ferromagnetic interlayer coupling between the magnetic layers in the SL as previously demonstrated for similar SLs by means of neutron diffraction measurements [9]. The results presented in Fig. 1 also show that the SL exhibits a much wider hysteresis loop and a greater magnitude of the PHE with respect to those of the reference (Ga,Mn)As layer. The larger coercivity of SL, indicating that the magnetization rotation is more difficult in the SL than in the single (Ga,Mn)As layer, may result from the ferromagnetic interlayer coupling occurring in the SL. Another possible reason for the larger coercivity of SL may be a decrease in hole density in the magnetic layers [10], resulting from diffusion of holes to the GaAs spacer layers. The latter effect may also be responsible for the lower $T_{\mathrm{C}}$ in the SL with respect to the reference $(\mathrm{Ga}, \mathrm{Mn})$ As layer [6].

In conclusion, we have shown that the PHE can be very useful in the study of magnetic properties of ferromagnetic semiconductors and their structures. The PHE can be also used as a basis for a new type of a non-volatile memory cell, in which a bit of information is written magnetically and read electrically.

\section{References}

[1] C. Goldberg, R.E. Davis, Phys. Rev. 94, 1121 (1954).

[2] H.X. Tang, R.K. Kawakami, D.D. Awschalom, M.L. Roukes, Phys. Rev. Lett. 90, 107201 (2003).

[3] T. Dietl, H. Ohno, F. Matsukura, Phys. Rev. B 63, 195205 (2001).

[4] M. Sawicki, K.-Y. Wang, K.W. Edmonds, R.P. Campion, C.R. Staddon, N.R.S. Farley, C.T. Foxon, E. Papis, E. Kamińska, A. Piotrowska, T. Dietl, B.L. Gallagher, Phys. Rev. B 71, 121302(R) (2005).

[5] T. Wosiński, A. Mạkosa, J. Sadowski, O. Pelya, V. Osinniy, T. Figielski, F. Terki, C. Hernandez, S. Charar, J. Alloys Comp. 423, 248 (2006).

[6] J. Sadowski, R. Mathieu, P. Svedlindh, M. Karlsteen, J. Kanski, Y. Fu, J.Z. Domagała, W. Szuszkiewicz, B. Hennion, D.K. Maude, R. Airey, G. Hill, Thin Solid Films 412, 122 (2002).

[7] T. Figielski, T. Wosiński, A. Morawski, A. Mạkosa, J. Wróbel, J. Sadowski, Appl. Phys. Lett. 90, 052108 (2007).

[8] T.R. McGuire, R.I. Potter, IEEE Trans. Magn. 11, 1018 (1975).

[9] W. Szuszkiewicz, E. Dynowska, B. Hennion, F. Ott, M. Jouanne, J.F. Morhange, M. Karlsteen, J. Sadowski, Acta Phys. Pol. A 100, 335 (2001).

[10] S.J. Potashnik, K.C. Ku, R.F. Wang, M.B. Stone, N. Samarth, P. Schiffer, S.H. Chun, J. Appl. Phys. 93, 6784 (2003). 